\title{
A 2D Staggered Multi-Material ALE Code Using MOF Interface Reconstruction
}

\author{
Yibing Chen ${ }^{1}$, Junxia Cheng ${ }^{1, *}$, Liang Pan ${ }^{2}$, Haihua Yang ${ }^{1}$, \\ Heng Yong ${ }^{1}$ and $\mathrm{Xin} \mathrm{Yu}^{1}$ \\ ${ }^{1}$ Institute of Applied Physics and Computational Mathematics, Beijing, China \\ ${ }^{2}$ School of Mathematical Science, Beijing Normal University, Beijing, China
}

Received 13 May 2020; Accepted (in revised version) 3 August 2020

\begin{abstract}
Hydrocodes are necessary numerical tools in the fields of implosion and high-velocity impact, which often involve large deformations with changing-topology interfaces. It is very difficult for Lagrangian or Simplified Arbitrary LagrangianEulerian (SALE) codes to tackle these kinds of large-deformation problems, so a staggered Multi-Material ALE (MMALE) code is developed in this paper, which is the explicit time-marching Lagrange plus remap type. We use the Moment Of Fluid (MOF) method to reconstruct the interfaces of multi-material cells and present an adaptive bisection method to search for the global minimum value of the nonlinear objective function. To keep the Lagrangian computations as long as possible, we develop a robust rezoning method named as Combined Rezoning Method (CRM) to generate the convex, smooth grids for the large-deformation domain. Regarding the staggered remap phase, we use two methods to remap the variables of Lagrangian mesh to the rezoned one. One is the first-order intersection-based remapping method that doesn't limit the distances between the rezoned and Lagrangian meshes, so it can be used in the applications of wide scope. The other one is the conservative secondorder flux-based remapping method developed by Kucharika and Shashkov [22] that requires the rezoned element to locate in its adjacent old elements. Numerical results of triple point problem show that the result of first-order remapping method using ALE computations is gradually convergent to that of second-order remapping method using Eulerian computations with the decrease of rezoning, thereby telling us that MMALE computations should be performed as few as possible to reduce the errors of the interface reconstruction and the remapping. Numerical results provide a clear evidence of the robustness and the accuracy of this MMALE scheme, and that our MMALE code is powerful for the large-deformation problems.
\end{abstract}

AMS subject classifications: 65M10, 78A48

Key words: Staggered Lagrangian scheme, multi-material ALE, MOF interface reconstruction, large-deformation problems.

${ }^{*}$ Corresponding author. Email address: cheng-junxia@iapcm.ac.cn (J.X. Cheng) 


\section{Introduction}

Lagrangian hydrodynamic algorithms have been widely used for perhaps the longest time of numerical methods employed for the solution of the complex problem of multi-material fluid flows [8], because they can track the material interfaces clearly and avoid the numerical error of the advection. Moreover, it is easy for Lagrangian schemes to prescribe the boundary conditions, such as free boundary and moving pistons. Lagrangian hydrodynamics algorithms can be classified into the staggered Lagrangian and cell-centered Lagrangian algorithms. Cell-centered Lagrangian algorithms define physical variables and velocities at zone centers [27], which usually need to solve the Riemann problem on the cell boundaries. Staggered Lagrangian algorithms define physical variables of density, internal energy and pressure at zone centers, but velocities are defined at the nodes, which often use the artificial viscosity to stabilize the numerical scheme at the shock $[23,28]$, or else there will be violent oscillations with the solutions at shocks. Staggered Lagrangian schemes have the overwhelming advantages of being simple and inexpensive [12], so they have been widely used in applications for many years. However, Lagrangian algorithms have the intrinsic difficulty in tackling large-deformation problems with changing-topology interfaces, which can't be tackled by the improved Lagrangian algorithms because they were designed to tackle the unphysical deformations of Lagrangian mesh $[8,11,12]$.

Hirt et al. [18] put forward to the Arbitrary Lagrangian-Eulerian (ALE) method to integrate the advantages of Lagrangian and Eulerian methods. Prior to the paper [18], the computational fluid dynamicist was limited to Lagrangian and Eulerian methods [3]. Mesh in ALE scheme can move in the styles of Lagrangian, Eulerian or arbitrary providing additional flexibility and accuracy. However, this ALE method in reference [18] was a kind of single-material ALE, which was also called Simplified ALE (SALE) method, in other words, cells could and only could contain one material. Therefore, it could not rezone the mesh across the materials' interfaces, and so couldn't tackle the large-deformation problem with changing-topology interfaces. To overcome the numerical difficulties of large-deformation problems, multi-material ALE method has been developing in recent years, which has been implemented in such production hydrocodes as ALE3D [29], ALEGRA [33] and FLAG [24] etc.

There are two types of ALE methods: direct ALE and indirect ALE. Considering the mesh movements explicitly in the differential equations, direct ALE method has to solve the advection terms of the fluid equations in the form of ALE, the high-order direct ALE scheme can be developed more easily [19], but it is difficult in updating nonlinear physical variables which depend on the history such as stresses and strains. The indirect ALE method is called also as three-stage ALE or split ALE, which is comprised of three stages: (1) Lagrangian stage, in which the solution is updated, and nodes move in Lagrangian style; (2) Rezoning stage, in which the nodes of Lagrangian mesh move to more optimal positions to improve the quality of the mesh; (3) Remapping stage, in which the Lagrangian solution on the Lagrangian mesh is remapped onto the rezoned mesh. Because of the splitting operation, the indirect ALE method is more compatible 
with the Lagrangian method, and it is complemented more easily based on the existed Lagrangian codes, therefore it is extensively employed in production codes. In this paper, our MMALE code is also developed in the frame of the indirect ALE method based on the existed staggered SALE code.

Multi-material ALE method coupled to MOF method is comprised of the following four parts: closure model, interface reconstruction method, mesh rezoning method and variables remapping method. For multi-material cells, the closure model must be demanded to distribute the change in total volume of the multi-material cell between material components, update internal energy of each material component and common pressure of multi-material cells. The closure model has been an open problem until now $[3,25]$, and is still developing to obtain the more physical models recently $[1,2]$. The challenge of the closure model is to define the state of mixtures despite the lack of information about the velocity distribution within multi-material cells. By introducing some kinds of different assumptions, multiple closure models have been developed, for example, equal volumetric strain, pressure balanced/pressure relaxation [14], contact closure models [34], IA-SSD models $[1,4]$ etc. In all closure models, the closure model of equal volumetric strain is the simplest and employed extensively in application codes, which assumes that all the materials have the same compressibility, in other words, the volume fractions of multi-material cells don't change in time during Lagrangian step. Although it is simple and unphysical, it can give the acceptable results in many conditions. Therefore, we choose the closure model of equal volumetric strain as a starting point to develop our MMALE code, and the improvements will be made gradually afterwards according to the requirements of applications.

ALE methods based on pure material polygons have to reconstruct the material polygons in multi-material cells. Modern interface reconstruction methods use linear representations for the material interfaces. Volume of Fluid (VOF) method has been used extensively in Eulerian codes for several decades [30,32]. When volume fractions of object multi-material cell and its neighbors are known, VOF method can give the interfaces of the multi-material cell. However, when cells containing more than two materials, VOF method will give the different results according to the material order [13], and has to introduce other rules to give the material ordering [7]. MOF method was developed in recent years [13], which could naturally reconstruct the interfaces of multi-material cells containing more than two materials. MOF method needs the reference centroids of all materials besides their volume fractions in multimaterial cells. MOF method overcomes the difficulty of VOF method in the condition of more than two materials by minimizing the distances between the polygons' and the reference centroids. Furthermore, MOF performs more accurately to reconstruct the interfaces in multi-material problems [21]. Therefore, we choose the MOF method to reconstruct the interfaces of multi-material cells in this paper.

Rezone phase is to improve the quality of the mesh after Lagrangian stage using all kinds of grid generation methods or optimization methods. When MMALE technique is complemented, the challenges of rezoning methods are reduced to a great extent, but the robust rezoning methods is still desired to decrease the operation times of 
MMALE as much as possible. In our applications, an Optimization-Based Rezoning (OBR) method developed by Chen [10] has been used extensively to structured and unstructured mesh. Based on OBR method and variational Winslow grid generator [9], we have developed the Combined Rezonging Method (CRM), which is comprised of three steps: initialization, untangling and homogenization. CRM method is capable of generating the convex, smooth and homogeneous mesh for almost all conditions in our applications.

Remapping phase is the last step of MMALE, which is to remap variables of the Lagrangian mesh onto the rezoned mesh. Remapping methods are classified into intersection-based and flux-based methods. Flux-based methods usually are higher efficient compared with the intersection-based methods, but they usually require the closer distances between the rezoned and Lagrangian meshes. Therefore, flux-based methods will need the specialized rezoning methods in order to assure the close of the new mesh to the old one. Intersection-based methods need to obtain the intersections of rezoned and Lagrangian mesh, so they have much more searching work than fluxbased methods, but they can be used in applications of much wider scope and there is no special requirement to the rezoning methods. For the staggered schemes, kinematic and physical variables have different control volume, so it is difficult for the remapper to conserve the total energy. Kucharik and Shashkov [22] developed the conservative multi-material remap method for staggered MMALE methods, which distributed the discrepancy of the kinetic energy to the internal energy of its adjacent cells to conserve the total energy. In this paper, we complement both the first-order intersection-based remapping method and flux-based second-order conservative remapping method [22], and compare them in the numerical examples.

This paper is arranged as follows. Section 2 introduces key elements of the multimaterial Arbitrary Lagrangian-Eulerian scheme, including the traditional Lagrangian scheme, the MOF interface reconstruction method, the rezoning method and intersection-based Remapping method on quadrilaterals. Numerical results are given in Section 3. Section 4 gives the conclusions and perspectives about future works.

\section{Multi-material arbitrary Lagrangian-Eulerian (MMALE) scheme}

We will present the MMALE scheme by the descriptions of Lagrangian scheme, interface reconstruction method, rezoning method and remapping method. The flowchart of the MMALE code is shown in Fig. 1, which is comprised of Lagrangian scheme, SALE and MMALE components. Existed SALE algorithm is the good foundation of developing MMALE. To some extent, MMALE can reuse some components of SALE, for example, rezoning methods cooperating with the intersection-based remapper, some modules of remapping method. The initialization stage consists in defining the distribution of all the physical variables over the initial grids. During the Lagrangian phase, the gas dynamics equations are solved using a staggered moving mesh method, refer to Section 2.1. We proceed to the interface reconstruction using the MOF method, refer to Section 2.2. The improvement of the quality of the Lagrangian grid is performed by 


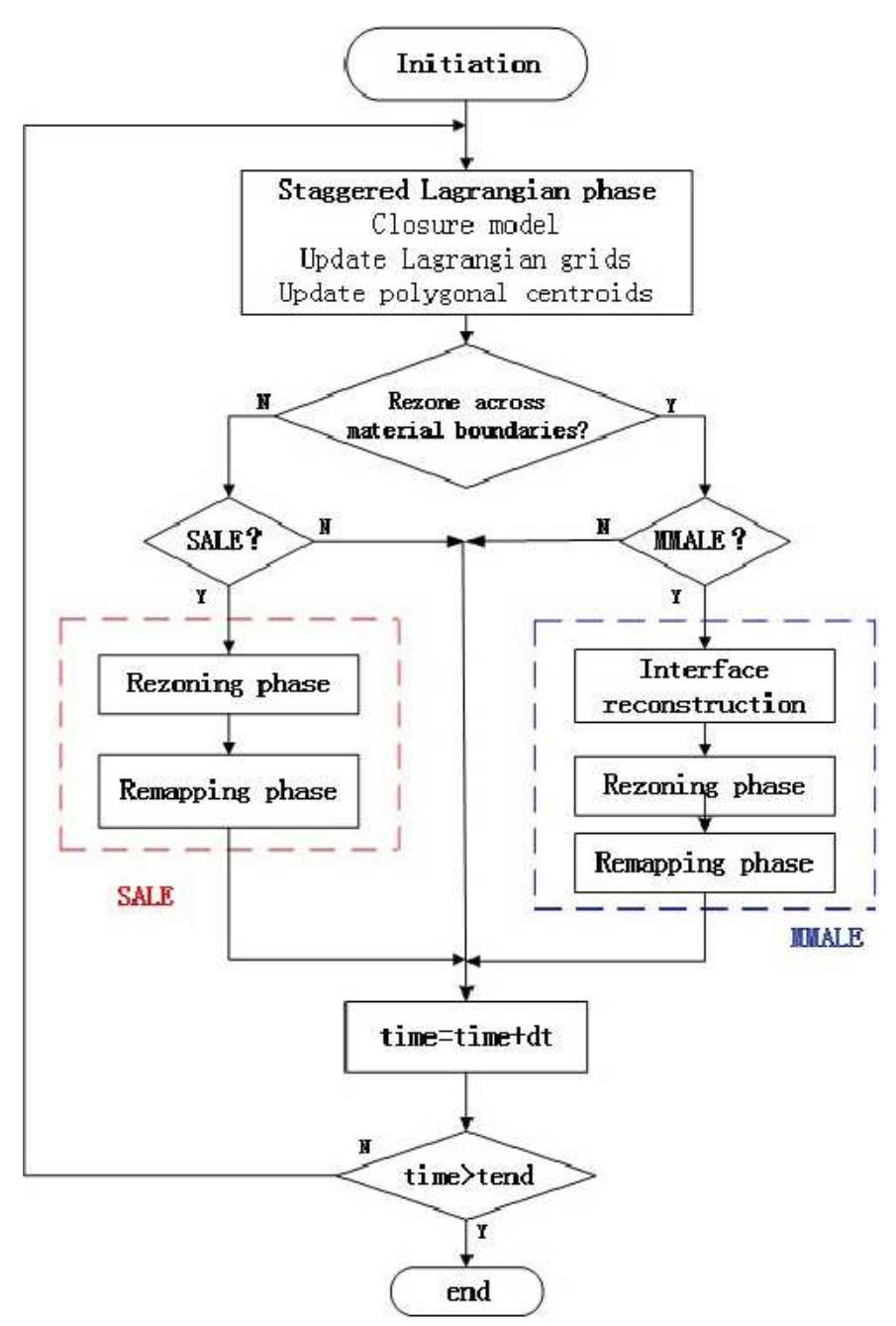

Figure 1: Flowchart of the staggered MMALE algorithm.

means of the rezoning phase, refer to Section 2.3. Finally, in the remapping stage, we remap all the variables of the Lagrangian mesh onto the new rezoned mesh, refer to Section 2.4. Two approaches are available: the first-order intersection-based remapping and the second-order flux-based remapping methods. The former can be used in arbitrary movements of the rezoned mesh while the latter is employed only in Eulerian regime in this paper.

\subsection{The traditional staggered Lagrangian scheme}

The Lagrangian phase consists of updating volume, momentum and internal energy of the control volume, assuming that the control volumes move in Lagrangian style. 
Eqs. (2.1) are the two-dimensional Lagrangian equations of the cylindrical coordinates.

$$
\left\{\begin{array}{l}
\dot{\rho}=-\rho\left(\frac{\partial u_{r}}{\partial r}+\frac{\partial u_{z}}{\partial z}+\alpha \frac{u_{r}}{r}\right) \\
\rho \dot{u}_{r}=-\frac{\partial p}{\partial r} \\
\rho \dot{u}_{z}=-\frac{\partial p}{\partial z} \\
\rho \dot{e}=-p \frac{d v}{v d t}
\end{array}\right.
$$

where $\rho, u_{r}, u_{z}, v, e, p$ are respectively the density, the velocity in the r-direction, the velocity in the z-direction, the specific volume, the specific internal energy and the pressure. When $\alpha=1$, Eqs. (2.1) are the corresponding equations of axisymmetric problems; when $\alpha=0$, they are the corresponding ones of the two-dimensional plane strain problem. In the Lagrangian scheme, the mass equation is naturally satisfied because the cell mass remains constant, shown in Eq. (2.2)

$$
\frac{d}{d t} \int_{V(t)} \rho d V=0
$$

where $V(t)$ is the Lagrangian control volume. The internal energy equation is discretized by the finite volume method, shown in Eq. (2.3)

$$
\int_{t^{n}}^{t_{n+1}} \int_{V(t)} d E d t=-\int_{t^{n}}^{t_{n+1}} \int_{V(t)} p d V d t
$$

where $E$ are the internal energy of the control volume. The central temporal difference rule is used to Eq. (2.3), which is discretized into Eq. (2.4)

$$
E^{n+1}-E^{n}=-\frac{1}{2}\left(p^{n}+p^{n+1}\right)\left(V^{n+1}-V^{n}\right),
$$

which should be combined with the equation of state to update the internal energy.

The momentum equations of the Lagrangian equations are discretized spatially by the isoparametric Galerkin bilinear finite element method shown in reference [5, page 42-51]. Lagrangian step uses the explicit central difference stepping method in time scheme, which is used in most hydrocodes [5,6]. Artificial viscosity has to be also used to stabilize the numerical scheme near the shock. The bulk viscosity used in this paper consists of the viscosity of von Neumann and Richtmyer [28] and the linear term added by Landshoff [23]. The hourglass viscosity should be used to suppress the nonphysical hourglass deformation, which is the consequence of using constant pressure quadrilateral elements $[5,11]$. 


\subsection{Moment Of Fluid (MOF) interface reconstruction method}

Similar to VOF, MOF interface reconstruction algorithm [13] uses linear interface to separate the materials in a multi-material cell. Dissimilarly, MOF method needs to know the centroids besides the volume fractions of materials in the multi-material cells. The centroids, updated in every Lagrangian step, are produced by closure model or result from the assumption of constant centroid positions in the parametric spaces [7]. When the volume fractions and centroids of each material in multi-material cells are known, the MOF method is used to obtain the polygons of pure materials by minimizing the distance between the reference and the polygonal centroids obtained as a result of reconstruction, subject to matching the given volume exactly. The distance function is shown as follows:

$$
g(\varphi)=\sum_{i}\left|\mathbf{x}_{i}^{c}(\varphi, d)-\mathbf{x}_{i}^{*}\right|, \quad \text { when } \quad V_{i}=V_{i}^{*}
$$

where $V_{i}^{*}$ is the given volume of material $i$ in the multi-material cell, $\mathbf{x}_{i}^{c}$ is the coordinate vector of the reconstructed polygon of material $i, \mathbf{x}_{i}^{*}$ is the coordinate vector of the given reference centroid of material $i, d$ is the signed distance of the interface to ensure $V_{i}=V_{i}^{*}$ when $\varphi$ is given, illustrated in Fig. 2.

For the multi-material cell containing two materials, when the normal of the interface is given, the material's polygon can be reconstructed at the certain signed distance while matching the specified volume. We should find the global minimum value of the objective function $g(\varphi)$ in the scope of $\varphi \in[0,2 \pi]$, and its corresponding normal should

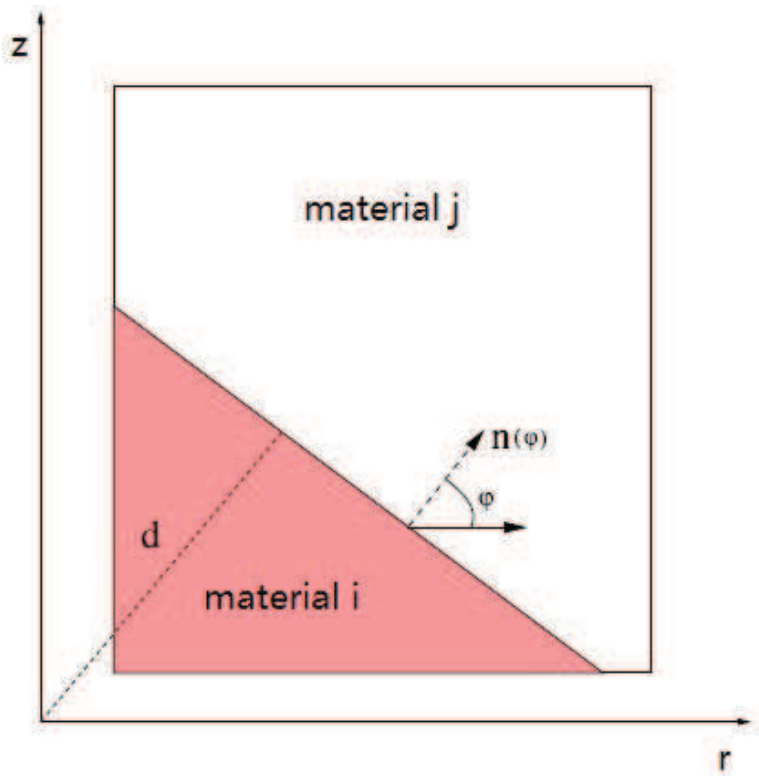

Figure 2: Illustration of definitions of the MOF method, where the half plane is defined by the angle $\varphi$ between its outward normal and the r-axis and the signed distance $d$. 


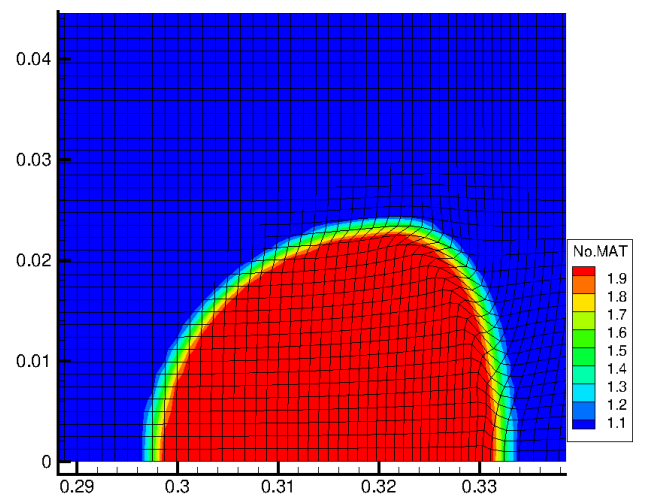

a) Material distribution weighted by volume fractions

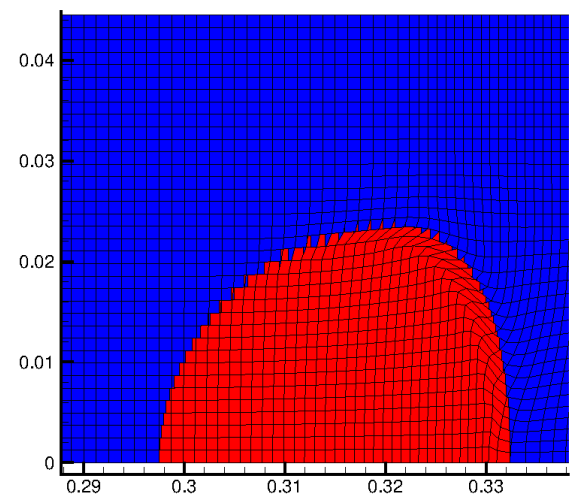

b) Reconstructed interfaces

Figure 3: Interface of bubble interacting with shock given by MOF method of traditional bisection method, where the material with red color is the bubble, and the other part with blue colored is the air.

be the one of the interface of multi-material cell. $g(\varphi)$ is the nonlinear function of $\varphi$, and it may have multiple local minima, so it is a challenge for the iteration methods to find the global minimum. Both the Newton-type and Brent iteration method need to compute the derivative of $g(\varphi)$, which has not the explicit expression, and the solution may depend on the initial value of $\varphi$ to a great extent. Moreover, the traditional bisection algorithm may also give the incorrect global minimum when $g(\varphi)$ has multiple minima. If the global minimum can't be found correctly, the reconstructed interfaces will be incorrect. For example, the traditional bisection algorithm can't correctly reconstruct the bubble on the general quadrilateral mesh, shown in Fig. 3(b), where the left part of the bubble is not loaded yet by the shock moving from the right, so it should be almost the shape of circular arc just like the shape shown in Fig. 3(a), which is the distribution of material number weighted by the volume fractions.

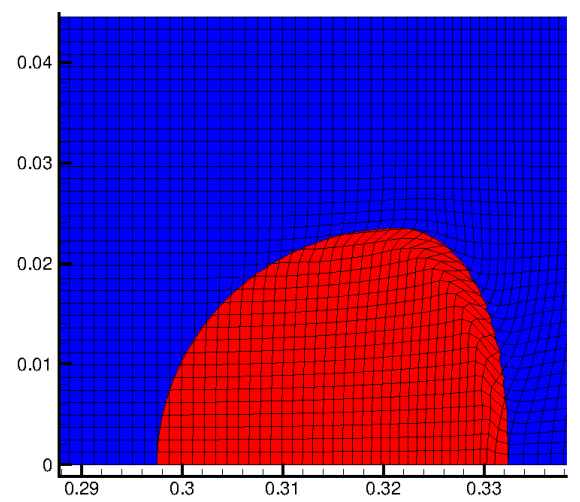

Figure 4: Interface of bubble interacting with shock given by MOF method of the adaptive bisection method, where the red-colored material is the helium bubble, and the other blue-colored part is the ambient air. 
Here, we develop an adaptive bisection algorithm - cf. Algorithm 2.1 below, which can find the global minimum and needs not to solve the derivative of the objective function.

\section{Algorithm 2.1}

1. Partition the region of $[0,2 \pi]$ into $n$ subregions, and get $g_{i}$ from Eq. (2.5) when $\varphi_{i}$ is given, subject to matching the given volume exactly. Note that $g_{1}=$ $g\left(\varphi_{1}=0\right), g_{2}=g\left(\varphi_{2}=\frac{2 \pi}{n}\right), \ldots, g_{n+1}=g\left(\varphi_{n+1}=2 \pi\right)=g_{1}$.

2. For $i=1, \ldots, n+1$, when $g_{i}<\min \left(g_{i-1}, g_{i+1}\right)$ is satisfied, function $g(\varphi)$ may have one or more than one minima in the scope of $\varphi \in\left[\varphi_{i-1}, \varphi_{i+1}\right]$, so number $i$ is stored in the array of $\aleph_{j}$. It is assumed that there are $m$ local minima initially.

3. For $j=1, \ldots, m$, search for the local minimum values of $g(\varphi)$ in the scope of $\left[\varphi_{\aleph_{j}-1}, \varphi_{\aleph_{j}+1}\right]$. Do the following loop for every element of $\aleph_{j}$, starting from $j=1$ :

(a) Initialize the parameters: $x_{l}=\varphi_{\aleph_{j}-1}, x_{h}=\varphi_{\aleph_{j}+1}, x_{m}=\varphi_{\aleph_{j}}, \omega=\frac{1}{2}\left(x_{h}-\right.$ $\left.x_{l}\right)$.

(b) Obtain the objective functions of $g\left(x_{l}\right), g\left(x_{h}\right), g\left(x_{m}\right)$ using Eq. (2.5).

(c) If $g\left(\varphi_{x_{m}}\right)>\max \left(g\left(x_{l}\right), g\left(x_{h}\right)\right)$, there may exist the local minimum values between $\left[x_{l}, x_{m}\right]$ and $\left[x_{m}, x_{h}\right]$, so $x_{l}, x_{h}$ should be added to the array of $\varphi_{i}$ and the element number of array $\aleph$ increases by two.

(d) Else if $g\left(x_{m}\right)<\min \left(g\left(x_{l}\right), g\left(x_{h}\right)\right)$, then $x_{l}=\frac{1}{2}\left(x_{l}+\omega\right), x_{h}=\frac{1}{2}\left(x_{h}+\omega\right), \omega=$ $\frac{1}{2} \omega$. If $\left|x_{l}-x_{h}\right|>\epsilon$, where $\epsilon$ is the given tolerance, then go to (b), or else go to $(\mathrm{g})$.

(e) Else if $g\left(x_{m}\right)<g\left(x_{h}\right)$, then $x_{h}=x_{m}, x_{m}=\frac{1}{2}\left(x_{h}+x_{l}\right), x_{l}=x_{l}-\omega$, go to (b).

(f) Else if $g\left(x_{m}\right)<g\left(x_{l}\right)$, then $x_{l}=x_{m}, x_{m}=\frac{1}{2}\left(x_{h}+x_{l}\right), x_{h}=x_{h}+\omega$, go to (b).

(g) $j=j+1$, if $j>m$, then go to step 4, or else go to (a).

4. Get the minimum of $g\left(\varphi_{\aleph_{j}}\right), j=1, \ldots, m$, and its corresponding angle is the one between the outward normal of the interface and r-axis.

Generally, $n=10$ is enough in most conditions. This adaptive bisection method does not depend on the initial value and needs not to compute the derivative of objective function, moreover, it can always find the global minimum value even when multiple minima exist. Fig. 4 is the result obtained by the adaptive bisection algorithm, 


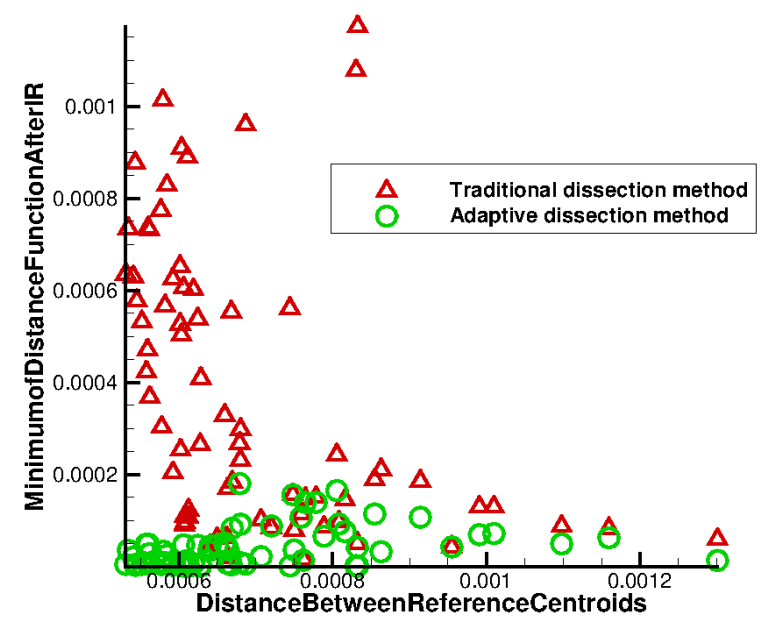

Figure 5: Comparison of the distances between the computed and reference centroids by MOF method of the traditional bisection and the adaptive bisection method. The lateral axis is the centroids' distances before reconstructing, and longitudinal axis is the centroids' distances after reconstructing.

where the left part of the bubble's interfaces is almost circular arc just like the shape it should be. The distance discrepancies between the reference and polygonal centroids are compared in Fig. 5, from which it can be seen that discrepancies by MOF employing the adaptive bisection method are much smaller than those by MOF employing the traditional bisection method. Fig. 5 shows that the traditional bisection method could not find the global minimum value for sure in many conditions.

When the multi-material cell contains more than two materials, interfaces can be reconstructed by different material orders, and the solution is chosen corresponding with the minimum distance between the reference centroids and the polygonal centroids. For the multi-material cell containing three materials, the interfaces can be reconstructed in the following steps. Firstly, material 2 and 3 can be combined as one new material called material 2-3, and reconstructed the interface between material 1 and material 2-3; secondly, after the polygon of material 2-3 is obtained, then reconstruct the interface between material 2 and material 3; thirdly, obtain the distance $g_{1-2-3}$ between the reference centroids and the polygonal ones of three materials, we denote this material order as (1-2-3). Similarly, repeat the above three steps to reconstruct the interfaces in the material order (2-1-3) and (3-1-2), and obtain $g_{2-1-3}, g_{3-1-2}$. Finally, choose the material order corresponding with the minimum value of $g_{1-2-3}, g_{2-1-3}, g_{3-1-2}$. It is similar to reconstruct the interfaces of the multimaterial cell containing more than three materials.

\subsection{Combined Rezoning Method (CRM)}

Rezoning phase is to obtain the higher-quality mesh by moving the Lagrangian nodes to the optimal positions. Rezoning methods should be chosen according to the 
remapping method to some extent. Intersection-based remapping method, which is used in this paper, does not constrain the movements of the nodes, so all the rezoning methods can be used. In extreme cases of large-deformation problems, such remesh method as Winslow method may generate concave grids and it will lead to the abortion of the computation. The variational form of the Winslow grid generator [9] was developed to overcome the difficulty of grid generation for large-deformation domains, which approximated the function itself of Eq. (2.6) rather than its Euler-Lagrange equations,

$$
I=\int\left[(\nabla \xi)^{2}+(\nabla \eta)^{2}\right] d x d y=\int \frac{1}{J}\left(x_{\xi}^{2}+y_{\xi}^{2}+x_{\eta}^{2}+y_{\eta}^{2}\right) d \xi d \eta
$$

where $J=x_{\xi} y_{\eta}-x_{\eta} y_{\xi}$ is the Jacobian of the mapping $x(\xi, \eta), y(\xi, \eta)$. The unconstrained minimization of Eq. (2.6) guarantees that all quadrilateral grid cells are convex at each iteration.

Chen and Jiang [10] presented an optimization-based rezoning method to improve the homogenization, orthogonality and smoothness of the mesh. Based on the work $[9,10]$, CRM method was developed later, which consisted of three steps: firstly, use some kind of simple grid generation method (Winslow method or transfinite interpolation method) to obtain the initial mesh, which can not guarantee that all the quadrilateral cells are convex; secondly, use variational Winslow grid method [9] to obtain the convex mesh, which guarantees the convexity of the mesh but cannot assure the smoothness and homogenization of the mesh; finally, use the Optimization-Based Rezoning (OBR) method to improve the smoothness, orthogonality and homogenization of the mesh, which is the solution of a constrained optimization problem [10]. CRM method is quite robust, and works for almost all the conditions in our applications. Fig. 6 shows that CRM gives comparable mesh with the result of the reference [9], therewith our result mesh has better smoothness and homogenization and the corner grids have the larger area. In addition, we have to add here that we cannot find the exact size of the reference result, so our numerical example (right figure in Fig. 6) may be almost the same but perhaps not exactly the same boundaries with the reference (left figure in Fig. 6).
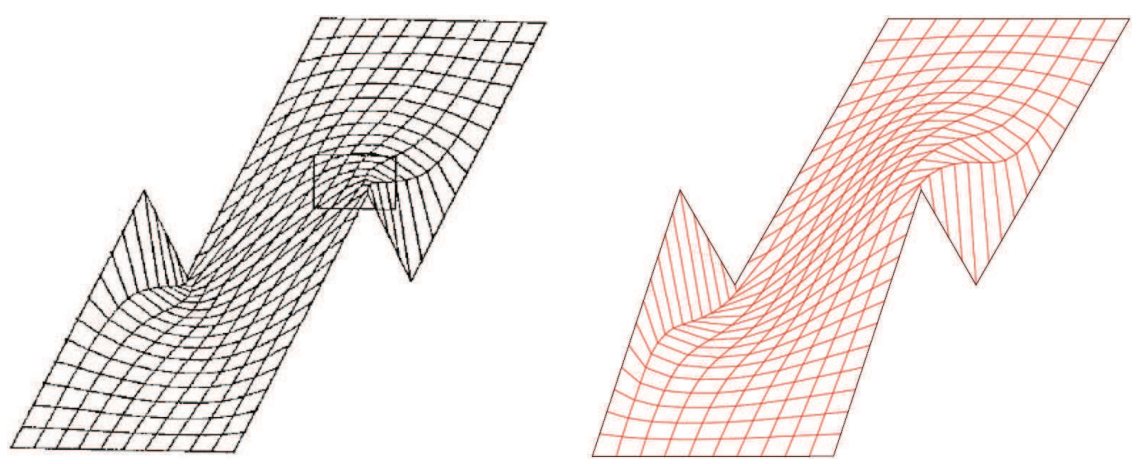

Figure 6: Meshes generated by the variational Winslow method (left) and the CRM method (right). 


\subsection{Remapping method}

Remapping phase is to remap all the variables of Lagrangian mesh onto the rezoned mesh including the zonal physical and nodal variables. In this paper, there are two kinds of remapping methods. One is the first-order intersection-based method, which does not constrain the mesh movement and can be used to the general conditions of arbitrary mesh movements. Cell-centered variables are constant on the cell, and the velocities are bilinear on the cells,

$$
u=\sum_{I=1}^{4} \phi_{I} u_{I},
$$

where $\phi_{I}=\frac{1}{4}\left(1+\xi_{I} \xi\right)\left(1+\eta_{I} \eta\right), \xi_{I}=[-1,1,1,-1], \eta_{I}=[-1,-1,1,1]$. When the remapping times are limited, it can give the acceptable results which will be seen in the following numerical examples. So, we use the first-order intersection-based method in this paper despite of its first-order accuracy when we want to decrease the frequency of rezoning as much as possible. The other remapper is the second-order flux-based remapping method [22] conserving the total energy and momentum, which is only used in Eulerian computations in this paper because of its limited movements of nodes. In this paper, the nodal momenta are remapped in slightly different ways with the reference [22] because of the different Lagrangian schemes. Here the element's mass is partitioned to its nodes equally instead of introducing the sub-zonal masses.

\section{Numerical results}

In the following examples, materials are some kinds of perfect gas, and their equations of state write $p=(\gamma-1) \rho e$, where $p$ is the pressure, $\gamma$ stands for the polytropic index of the gas, $e$ is the specific internal energy. ALE computations are performed using CRM rezoning method described in Section 2.3. Eulerian computation is performed as some kind of special ALE computation where nodes of the rezoned mesh is always back to their initial places. If the ALE computations are performed at the interval of 100 time steps, we will denote it by ALE100; we can also run auto-ALE computations, in other words, whether the mesh is rezoned or not is determined according to the quality of the mesh. The quality of quadrilateral cell is measured by its four internal angles and length ratios of opposite sides. In this paper, we set the critical internal angle and length ratio as $\omega_{c}=20^{\circ}, \iota_{c}=0.1$, respectively. When there is an internal angle is less than $20^{\circ}$ or greater than $160^{\circ}$, or the length ratio of opposite sides (the length of shorter side is divided by that of longer side) is less than 0.1, the ALE operation should be performed. Auto-ALE is efficient and practical because it is helpful to decrease the rezoning times as much as possible and reduce the error of remapping and interface reconstruction. 


\subsection{Incompressible Rayleigh-Taylor instability problem}

In the $\left[0, \frac{1}{3}\right] \times[0,1]$ computational domain, there are two kinds of immiscible fluids and the interface between them is disturbed whose equation writes $z_{i}(r)=0.5+$ $0.01 \cos (2 \pi r)$. The heavy fluid of $\rho_{h}=2$ is on the top of the light fluid of $\rho_{l}=1$, and they have the same polytropic index $\gamma=1.4$. The domain is covered by the mesh of $30 \times 100$ by the body-fitted mesh, in other words, there are no multi-material cells at the beginning. The fluid is at rest everywhere initially. The initial pressure can be deduced by the following equations [15]:

$$
\begin{array}{ll}
p_{h}(r, z)=1+\rho_{h} g_{z}(z-1), & \text { if } \quad z>z_{i}(r), \\
p_{l}(r, z)=1+\rho_{h} g_{z}\left[z_{i}(r)-1\right]+\rho_{l} g_{z}\left[z-z_{i}(r)\right], & \text { if } \quad z \leq z_{i}(r),
\end{array}
$$

where $g_{z}=-0.1, \rho_{l}=1, \rho_{h}=2$.

This configuration is unstable, and the heavy fluid will sink while the light fluid will rise when time evolves. Due to the initial cosinusoidal interface, instabilities develop in the vicinity of the interface and lead into the mushroom-like interface at later time. Although this problem is incompressible, we run it using the compressible MMALE code coupled to MOF interface reconstruction just like the reference [16]. We run this problem on the Eulerian mesh till $t=9$. As shown in Fig. 7, our results develop a little more rapidly comparing with the reference result [16] using VOF interface reconstruction. There are three main differences between our scheme and the reference one [16]: (1) different Lagrangian schemes: staggered scheme versus cell-centered scheme; (2) different interface reconstruction methods: MOF versus VOF, which will lead to the slight difference [15]; (3) different remapping methods: staggered secondorder conservative remapping method versus cell-centered second-order conservative remapping method. We think that the differences of results are mainly brought by the different Lagrangian schemes, and remapping method may contribute to some extent, but not too much.
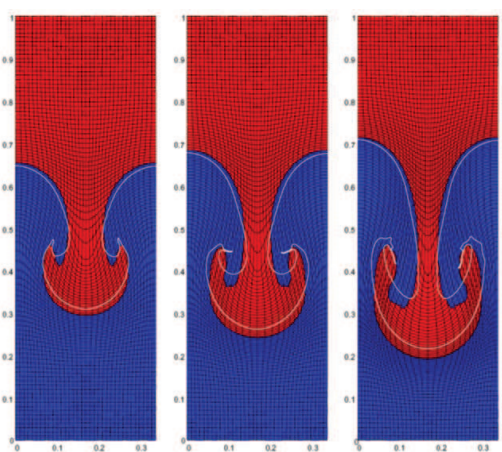

a) Reference results [16]

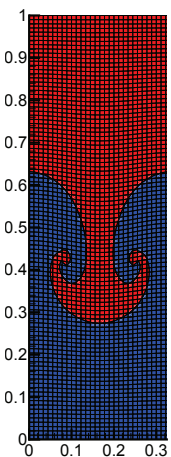

b) $t=7$

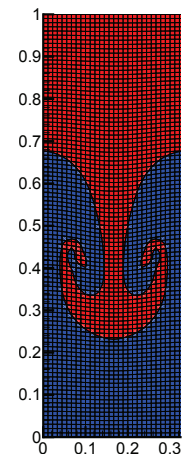

c) $t=8$

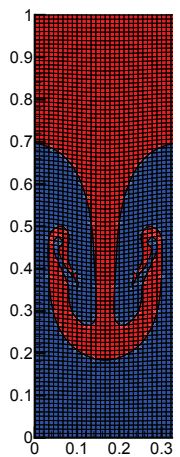

d) $t=9$

Figure 7: Comparison of the reference [16] and the results of this paper using second-order conservative remapping method at $t=7,8,9$. Different colors denote different materials. 

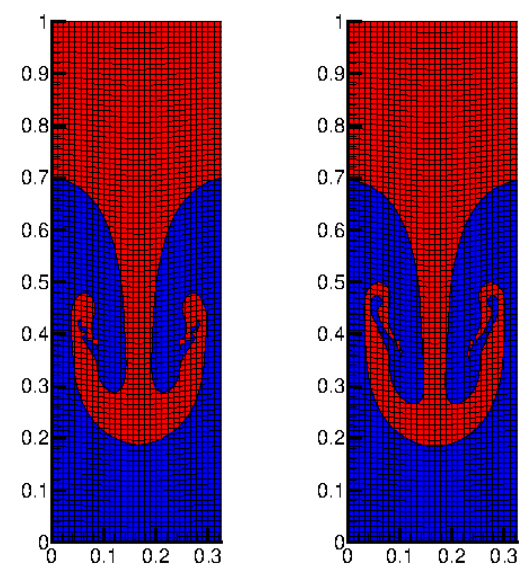

Figure 8: Comparisons of results obtained separately by the first-order intersection-based remmapping method (left) and the second-order flux-based remapping method (right) at $t=9$.

As shown in Fig. 8, results obtained by the second-order remapping method are quite similar to the ones by first-order remapping method considering 13265 rezoning times, and the instabilities of the former develop more quickly.

\subsection{Triple point problem}

A rectangular $[0,7] \times[0,3]$ domain is split into three sub-domains, shown in Fig. 9 . Material boundaries are initially aligned with the equidistant mesh containing $140 \times 60$ square cells. Left from $x=1$ line, a high-pressure material is located with the state of $(\gamma=1.5, \rho=1, p=1)$. The rest is split by the line of $y=1.5$ into two parts. The top half of the right domain is the light material with the state of $(\gamma=1.5, \rho=0.125, p=0.1)$, the bottom half is the heavy material with the state of $(\gamma=1.4, \rho=1, p=0.1)$, shown in Fig. 9.

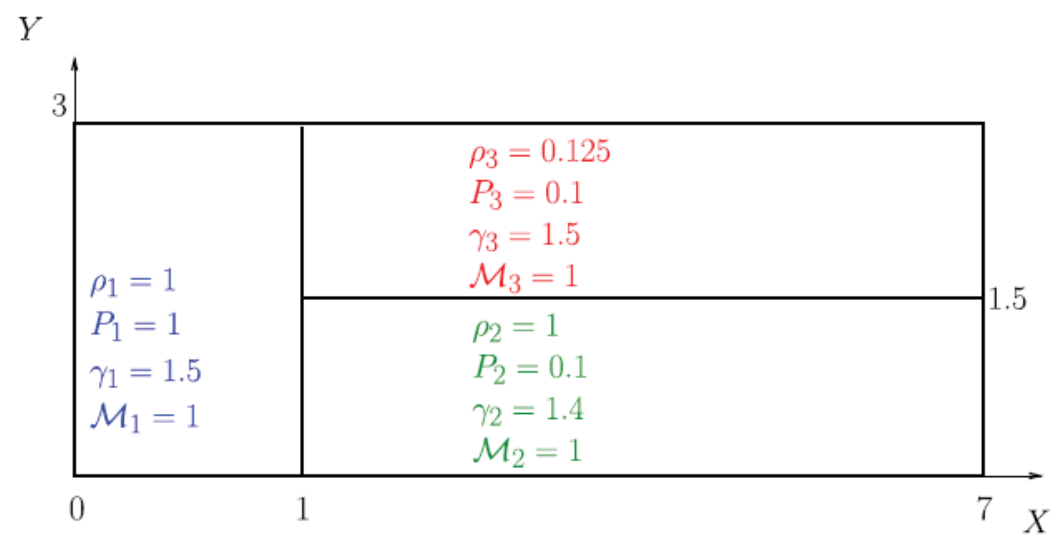

Figure 9: Initial condition of triple-point problem [15]. 


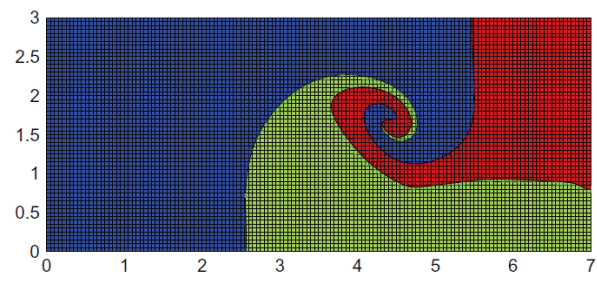

a) Reference result [15]

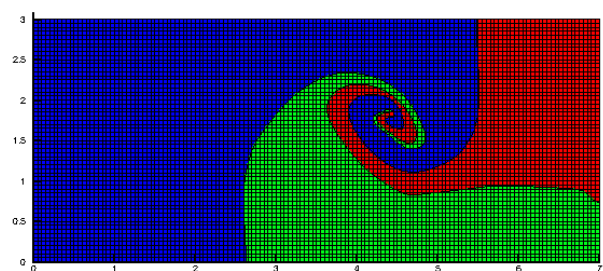

b) Result of this paper

Figure 10: Comparison of the reference and our results of triple-point problem in Eulerian computations at time $=5$ (the left is from the reference [15] and the right is the result of this paper obtained by the second-order remapping method).
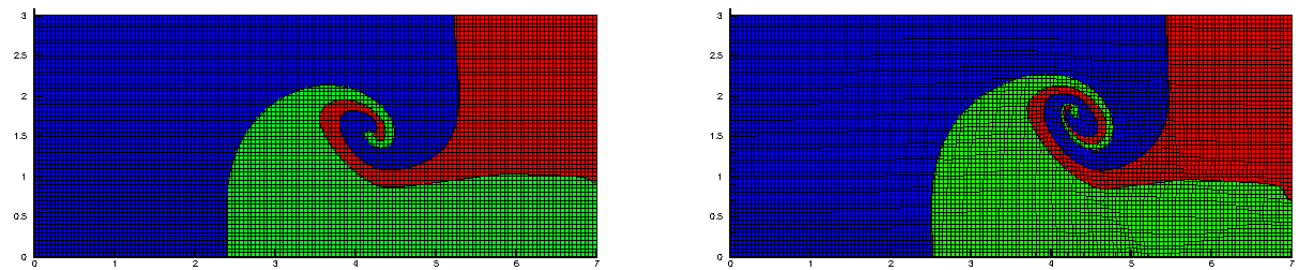

Figure 11: Comparison of results at time $=5$ separately obtained by the first-order remapping method in Eulerian computations (left) and in ALE100 regime (right).

In this problem, a shock wave propagates from the left to the right, a vortex is generated around the triple point because of different speeds of the right two materials.

We run this simulation on the Eulerian mesh till $t=5$. The material distribution in the final time is shown in Fig. 10. The left figure is the reference result in paper [15], and the right figure is our result. As we can see, our result is similar to the reference result except for the local place of the triple point, where the material remain reasonably compact and interfaces are smooth. The instability develops more rapidly in our result comparing with the reference one, which is sensitive to ALE times. As shown in Fig. 11, result obtained by the first-order remapping method in Eulerian regime is diffusive, but the result in ALE100 regime by the first-order remapping method is comparable with the result obtained by the second-order remapping method in Eulerian computations, and may be better in the local place of triple point. Results of Fig. 11 show that rezoning operations should be used as few as possible and Lagrangian computations should be kept as long as possible till the mesh is highly distorted, because both the remapping stage and interface reconstruction method always produce the error to some extent despite of whatever accuracy.

\subsection{Multi-material cylindrical shock-bubble interaction}

The shock-bubble interaction is a well-known problem, which has been extensively studied experimentally [17] and numerically [16, 22, 31,35]. Cylindrical shock-bubble interaction is a two-dimensional plane problem. The equidistant mesh of $520 \times 72$ cells 


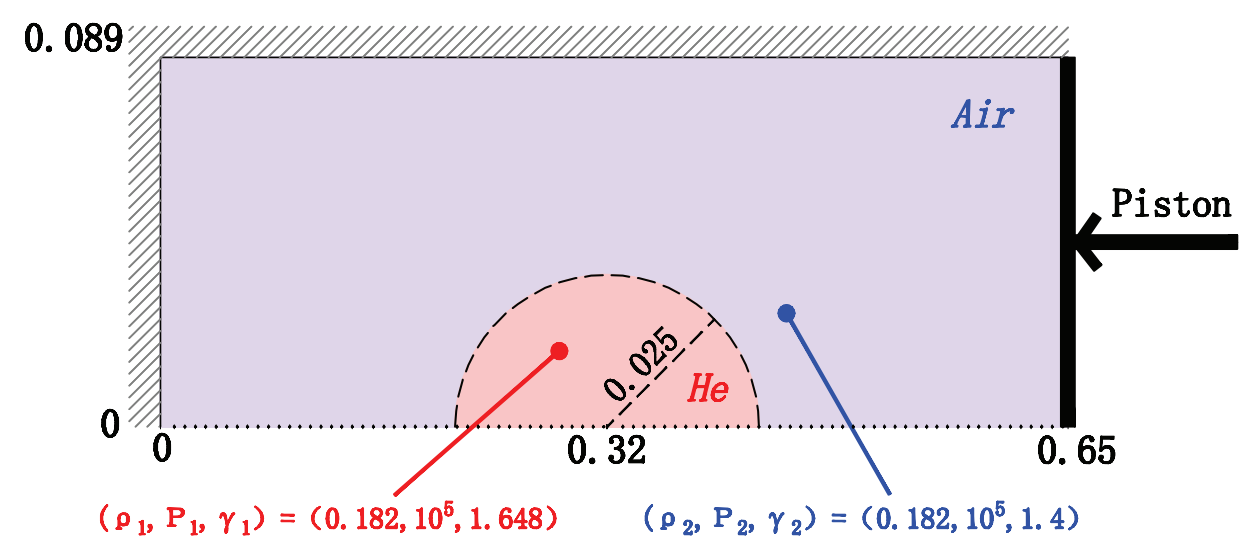

Figure 12: Initial condition of cylindrical Shock-bubble interaction problem.

covers the $[0,0.65] \times[0.0,0.089]$ domain. Initially, the fluid is at rest everywhere except for the right boundary, which is a piston moving with the velocity of $[-124.824,0]$ generating an $M_{s}=1.22$ shock wave propagating from the right to the left. The helium bubble is centered at $(0.32,0)$ with the radius of 0.025 , whose parameters are $\rho=0.182, p=10^{5}, \gamma=1.648$, and the rest of the domain is filled of air with the parameters of $\rho=1, p=10^{5}, \gamma=1.4$, shown in Fig. 12 .

The incident shock wave hits the bubble at the time of $668.153 \times 10^{-6}$, and at the time of $1342.153 \times 10^{-6}$ the shock wave hits the left domain boundary. The ALE200 simulation runs only in the local region where shock interacts with the bubble by the use of first-order intersection-based remapping method. Figs. 13-15 show that numerical results are in accord with the experimental results. Fig. 15 shows that our result is in good agreement with the numerical result of the reference paper [22], but there are some differences with the experimental result [17]. As shown in reference [31], there were also such differences between the numerical and experimental results.

\subsection{Multi-material spherical shock-bubble interaction}

This is a two-dimensional axi-symmetrical problem. This numerical example is performed on the $520 \times 36$ equidistant mesh covering all the domain. Initially, the fluid is at rest everywhere except for the right boundary, which is a piston moving with the velocity of $[-140.312,0]$ generating an $M_{s}=1.25$ shock wave propagating from the right to the left. The helium bubble is centered at $(0.32,0)$ with the radius of 0.0225 with parameters $\rho=0.182, p=10^{5}, \gamma=1.648$, and the rest of the domain is filled of air with the parameters of $\rho=1, p=10^{5}, \gamma=1.4$, shown in Fig. 16. The incident shock wave hits the bubble at the time of $657.463 \times 10^{-6}$. The ALE200 simulation runs in the local region where shock interacts with the bubble by the use of first-order intersection-based remapping method. Figs. 17-20 show that our numerical results are in quite good agreement with the experimental results [17]. 

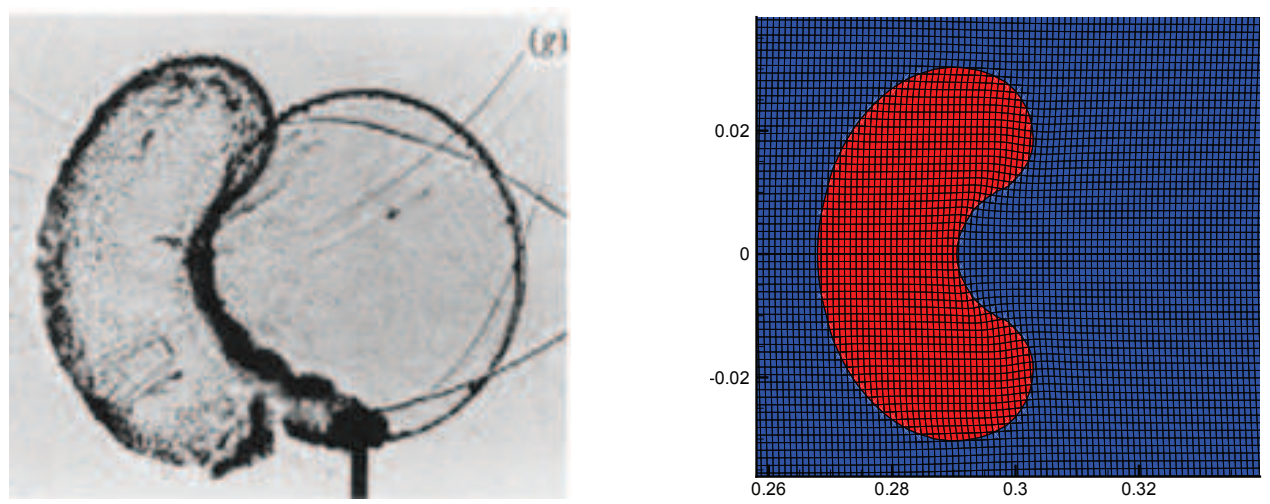

Figure 13: Cylindrical shock-bubble interaction problem at $t=913.153 \times 10^{-6}$, where the red-colored is the helium bubble and blue-colored is air. The left figure is the experimental result [17], and the right one is the numerical result of this paper.
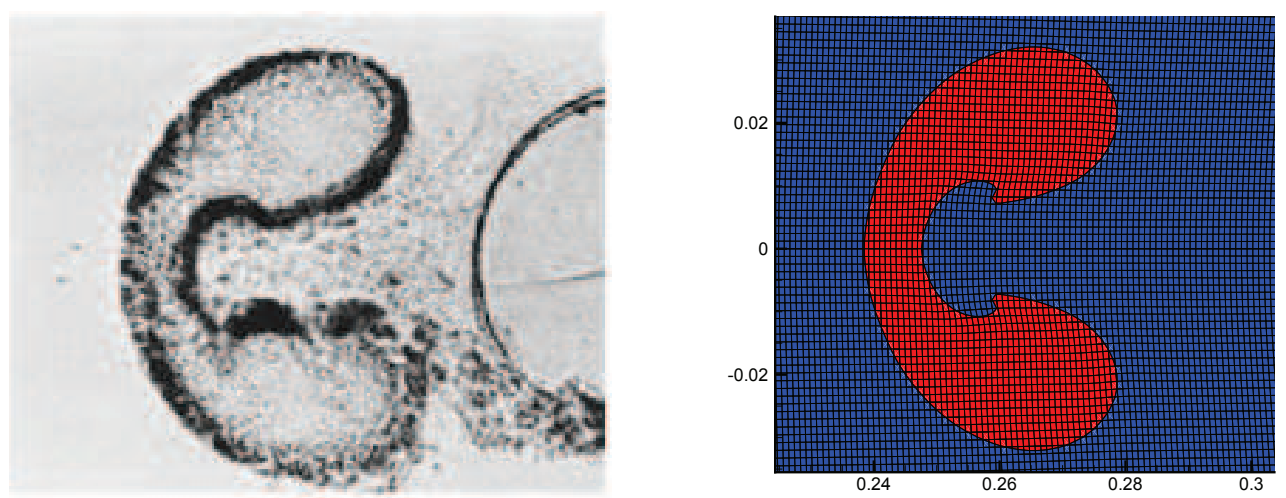

Figure 14: Cylindrical shock-bubble interaction problem at $t=1095.153 \times 10^{-6}$. The left figure is the experimental result [17], and the right one is the numerical result of this paper.
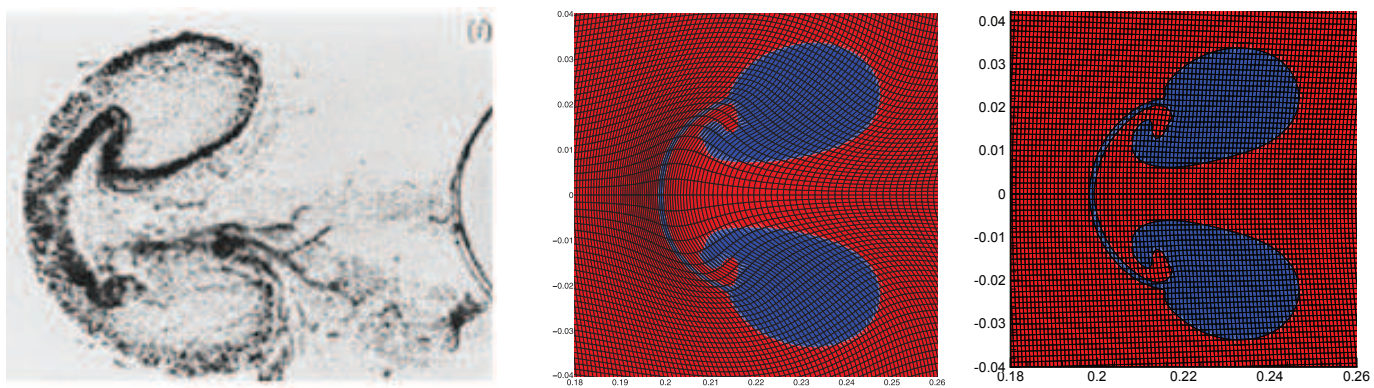

Figure 15: The density distribution for the multi-material cylindrical shock-bubble interaction problem at $t=1342.153 \times 10^{-6}$. Left figure is the experimental result [17], the middle one is the reference result [22] in the ALE10 regime, and the right one is our result in ALE200 regime, where the blue-colored part is helium bubble in the middle and right figure. 


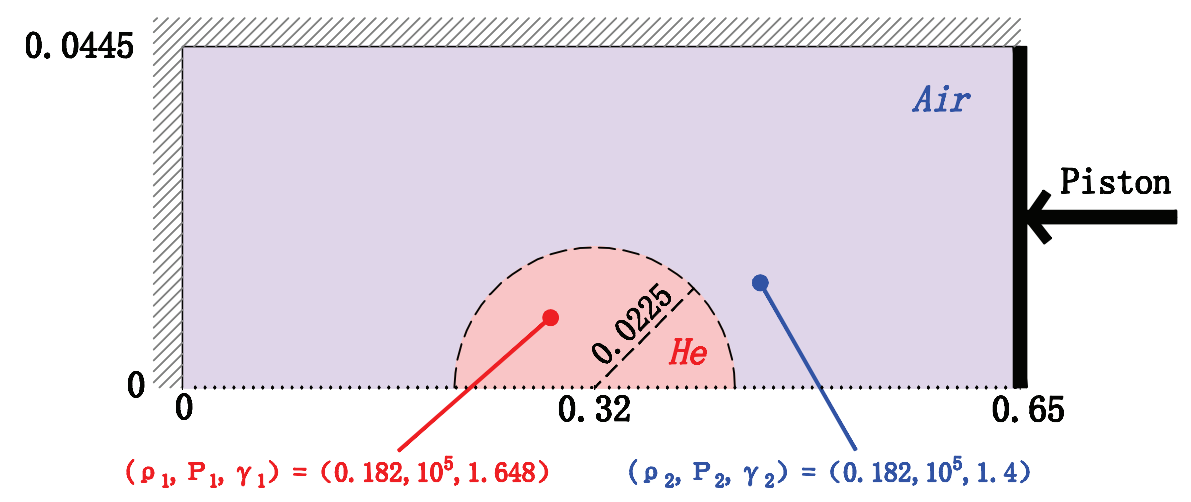

Figure 16: Initial condition of spherical shock-bubble interaction problem.
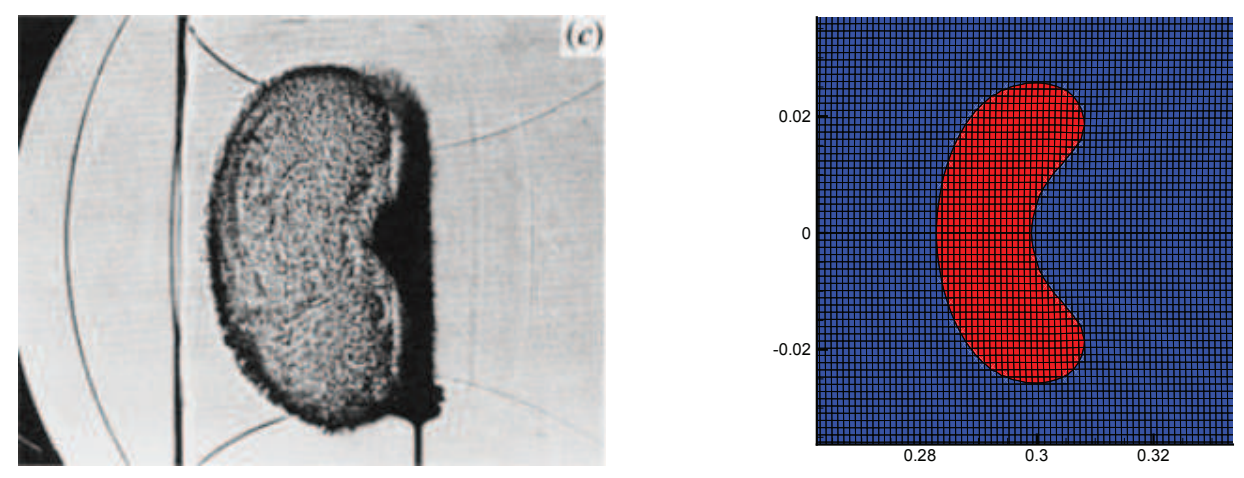

Figure 17: Spherical shock-bubble interaction problem at $t=802.463 \times 10^{-6}$. The left figure is the experiment result [17], the right one is our result which is the map of the material number.
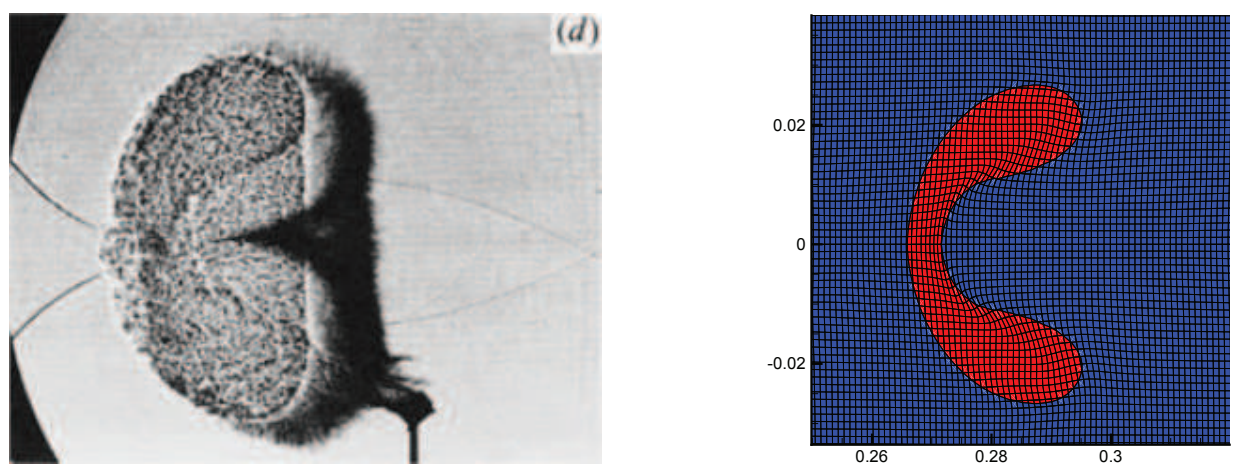

Figure 18: Spherical shock-bubble interaction problem at $t=880.463 \times 10^{-6}$. The left figure is the experiment result [17], the right one is our result which is the map of material number.

\subsection{Stratified Richtmyer-Meshkov instability problem}

The computational region is a rectangular box $[0,3] \times[0,1]$, which is paved with $300 \times 100$ cells. The disturbed interface is described by $r(z)=1.2+0.18 \cos (4 \pi z)$. 

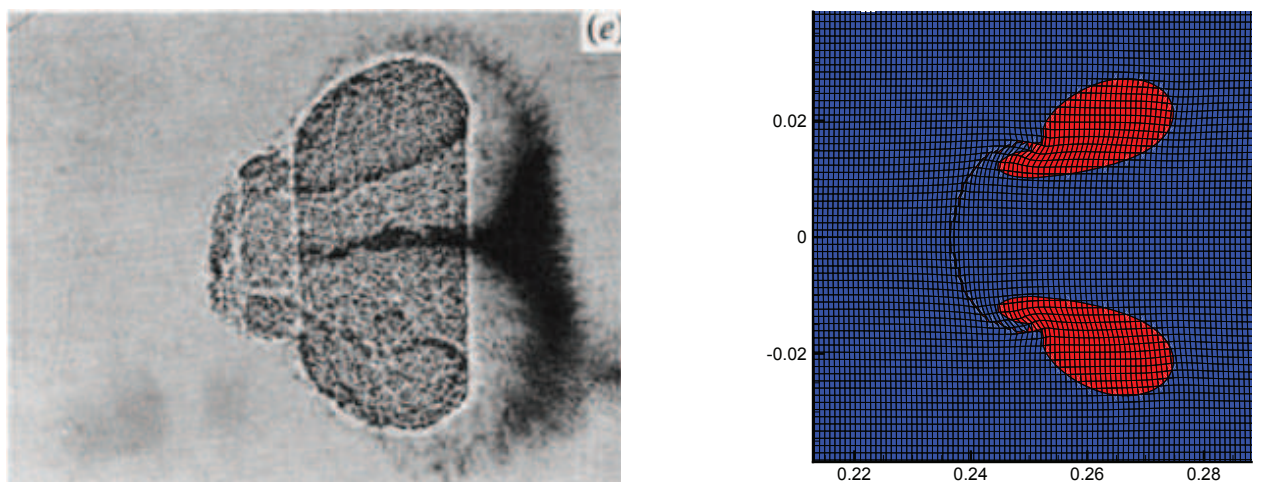

Figure 19: Spherical shock-bubble interaction problem at $t=1007.463 \times 10^{-6}$. The left figure is the experiment result [17], the right one is our result which is the map of material number.
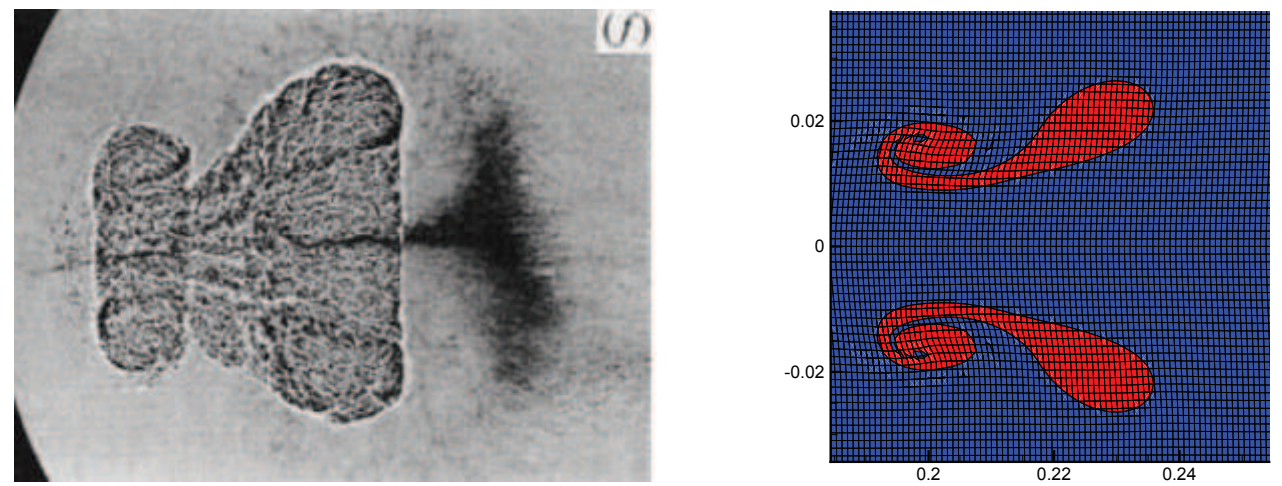

Figure 20: Spherical shock-bubble interaction problem at $t=1257.463 \times 10^{-6}$. The left figure is the experiment result [17], the right one is our result which is the map of material number.

The initial conditions are shown in Fig. 21, and all the boundaries are rigid walls. The initial mesh doesn't contain the multi-material cells. Initially the red-colored region of $r \in[0,0.3]$ is paved with the mesh $50 \times 100$ with the initial material state is $(\rho, \gamma, p)=$ $(3,1.4,12)$. The blue-colored neighborhood of the red-colored part is paved with $30 \times$ 100 cells characterized by $(\rho, \gamma, p)=(1,1.4,1)$. The green-colored part, starting from the line of $r=0.6$ to the disturbed interface, is paved with the mesh $58 \times 100$ with the initial material state $(\rho, \gamma, p)=(10,1.67,1)$. The right blue-colored part is paved with the mesh $162 \times 100$ that has the same initial material state with the left bluecolored material. The disturbed interface will be loaded by the shock moving from the left to the right. The initial two concave parts of the disturbed interface will be strengthened by the shock and leads to the formations of the mushroom-like shapes. Auto-ALE operations start from $t=0.4$. The two mushrooms are well developed and almost same at $t=1.5$, shown in Fig. 22. 


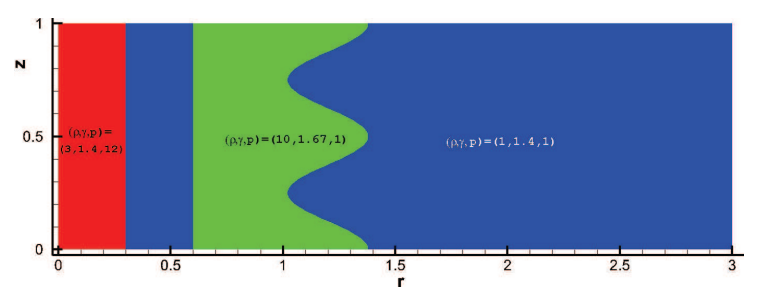

Figure 21: Initial data for the stratified Richtmyer-Meshkov instability problem.
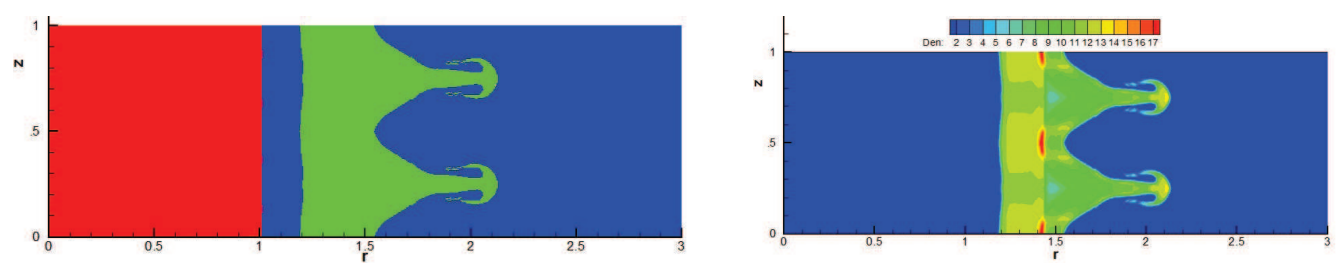

Figure 22: Numerical result of stratified Richtmyer-Meshkov instability problem at $t=1.5$ in auto-ALE regime (the left is the distribution of material, and the right is the density distribution).

\section{Conclusions}

We have presented a robust staggered multi-material ALE scheme coupled to MOF interface reconstruction, which is devoted to the numerical simulation of two-dimensional multi-material compressible large-deformation problems on quadrilateral grids with fixed topology. The MOF method using adaptive bisection method is presented to obtain the global minimum value of the nonlinear objective function for sure, which needs not to solve the derivative of objective function. We also develop the robust CRM rezoning method to generate convex-assured grids for the large-deformation region, which is helpful for Lagrangian computations to be performed as long as possible. The intersection-based remapping method is used because it is helpful to decrease the frequency of rezoning as much as possible. With the decrease of rezoning operations, the results obtained by the first-order intersection-based remapping method are closer gradually to the ones by the second-order remapping method in Eulerian computations. So, it is important for MMALE computations to decrease the frequency of the rezoning in order to reduce the errors of remapping phase and interface reconstruction. Numerical results of examples show that our MMALE code is robust and can give the acceptable results for large-deformation problems with limited rezoning times despite that the first-order remapping method is used.

In this paper, the intersection-based remapping method is only first-order, and the second-order flux-based conservative remapping method is used only in Eulerian computations to avoid the special grid generation method because of its limitation of distances between the rezoned and Lagrangian meshes. Therefore, we are developing the second-order conservative intersection-based remapping method in order to increase the accuracy of remapping method and decrease the frequency of rezoning as much as possible at the same time. Additionally, the equivalent stain closure model is used in 
this paper because it is simple and robust, and in the future we will work on the closure model starting with some existed ones such as IA-SSD $[1,4]$, pressure relaxation models [20] etc.

\section{Acknowledgments}

This work was performed under the auspices of National Natural Science Foundation of China (No. 11702030) and NSAF (No. U1630247).

The authors thank Professor Shuanghu Wang for his valuable instructions and helpful suggestions over the years, and mourn with deep grief for him.

\section{References}

[1] A. J. BARlow, R. Hill AND M. J. Shashkov, Constrained optimization framework for interface-aware sub-scale dynamics closure model for multimaterial cells in Lagrangian and Arbitrary Lagrangian-Eulerian hydrodynamics, J. Comput. Phys., 276 (2014), 92-135.

[2] A. J. BARLOW, M. KLIMA AND M. J. SHASHKov, Constrained optimization framework for interface-aware sub-scale dynamics models for voids closure in Lagrangian hydrodynamics, J. Comput. Phys., 371 (2018), 914-944.

[3] A. J. Barlow, P. -H. Maire, W. J. Rider, R. N. Rieben AND M. J. Shashkov, Arbitrary Lagrangian-Eulerian methods for modeling high-speed compressible multimaterial flow, J. Comput. Phys., 322 (2016), 603-665.

[4] A. J. Barlow, N. Morgan AND M. J. Shashkov, Constrained optimization framework for IA-SSD closure model for multimaterial cells in Lagrangian cell-centered hydrodynamics, Comput. Math. Appl., 78 (2019), 541-564.

[5] T. Belytschko, W. K. LiU AND M. Brian, Nonlinear finite elements for continua and structures, Zhuang Zhuo, Tsinghua University Press, Beijing, 2002.

[6] D. J. Benson, Computational methods in Lagrangian and Eulerian hydrocodes, Comput. Methods Appl. Mech. Engrg., 99 (1992), 235-394.

[7] D. J. BENSON, Volume of fluid interface reconstruction methods for multi-material problems, Appl Mech Rev, 55(2) (2002), 151-165.

[8] E. J. CARAmAna AND M. J. ShashKov, Elimination of artificial grid distortion and hourglass-type motions by means of Lagrangian subzonal masses and pressures, J. Comput. Phys., 142 (1998), 521-561.

[9] A. A. ChARAKHCH'YAN AND S. A. IVANENKO, A variational form of the Winslow grid generator, J. Comput. Phys., 136 (1997), 385-398.

[10] Y. B. Chen AND S. JIANG, An optimization-based rezoning for ALE methods, Commun. Comput. Phys., 4 (2008), 1216-1244.

[11] J. CHENG AND B. TIAN, Elimination of hourglass distortions by means of the LagrangianGauss-point-mass method for compressible fluid flows, Comput. and Fluids, 116 (2015), 148-157.

[12] J. K. Dukowicz And B. J. A. Meltz, Vorticity errors in multidimensional Lagrangian codes, J. Comput. Phys., 99 (1992), 115-134.

[13] V. DYADECHKO AND M. J. SHASHKOV, Reconstruction of multi-material interfaces from moment data, J. Comput. Phys., 227(11) (2008), 5361-5384. 
[14] M. M. Fransois, M. J. Shashkov, J. Fung, A. K. Harrison and T. R. Canfield, A comparative of multimaterial Langrangian and Euler methods with pressure relaxation, J. Comput. Phys., 83 (2013), 126-136.

[15] S. GALERA, J. BREIL AND P. -H. MAIRE, A $2 D$ unstructured multi-material cell-centered arbitrary Lagrangian-Eulerian (CCALE) scheme using MOF interface reconstruction, Comput. and Fluids, 46 (2011), 237-244.

[16] S. Galera, P.-H. Maire AND J. Breil, A two-dimensional unstructured cell-centered multimaterial ALE scheme using VOF interface reconstruction, J. Comput. Phys., 229 (2010), 5755-5787.

[17] J. F. HAAS AND B. STURTEVANT, Interaction of weak shock wave with cylindrical and spherical gas inhomogeneities, J. Fluid Mech., 181 (1987), 41-76.

[18] C. W. HiRT, A. A. AMSDEN AND J. L. CoOK, An arbitrary Lagrangian-Eulerian computing method for all flow speeds, J. Comput. Phys., 14(3) (1974), 227-253.

[19] P. Jin, X. DENG AND F. XIAO, A direct ale multi-moment finite volume scheme for the compressible euler equations, Commun. Comput. Phys., 24 (2018), 1300-1325.

[20] J. R. Kamm, M. J. ShashKov, J. Fung, A. K. HarRison AND T. R. CANField, A comparative study of various pressure relaxation closure models for one-dimensional two-material Lagrangian hydrodynamics, Internat. J. Numer. Methods Fluids, 65(12) (2011), 13111324.

[21] M. Kucharik, R. V. Garimella, S. P. Schofield and M. J. Shashkov, A comparative study of interface reconstruction methods for multi-material ALE simulations, J. Comput. Phys., 229(7) (2010), 2432-2452.

[22] M. Kucharik And M. J. Shashrov, Conservative multi-material remap for staggered multi-material arbitrary Lagrangian-Eulerian methods, J. Comput. Phys., 258 (2014), 268304.

[23] R. LANDSHOFF, A numerical method for treating fluid flow in the presence of shocks, Technical Report LA-1930, 1955.

[24] D. MANDELL, D. BURTON AND C. Lund, High explosive programmed burn in the FLAG code, Technical Report LA-113406, 1998.

[25] L. G. MARGOLIN, Introduction to an arbitrary Lagrangian-Eulerian computing method for all flow speeds, J. Comput. Phys., 135 (1997), 198-202.

[26] P.-H. MAIRE, A high-order cell-centered Lagrangian scheme for compressible fluid flows in two-dimensional cylindrical geometry, J. Comput. Phys., 228 (2009), 6882-6915.

[27] P.-H. MAIRE, R. AbGRALL, J. BREIL AND J. Ovadia, A cell-centered Lagrangian scheme for two-dimensional compressible flow problems, SIAM J. Sci. Comput., 29(4) (2007), 17811824.

[28] J. VOn NEUmann AND R. D. Richtmyer, A method for the numerical calculation of hydrodynamic shocks, J. Appl. Phys., 21 (1950), 232-237.

[29] C. Noble, A. Anderson, N. Barton, J. Bramwell, A. Capps, M. Chang, J. Chou, D. Dawson, E. Diana, T. Dunn, D. FauX, A. Fisher, P. Greene, I. Heinz, Y. Kanarska, S. Khairallah, B. Liu, J. Margraf, A. Nichols, R. Nourgaliev, M. Puso, J. Reus, P. Robinson, A. Shestakov, J. Solberg, D. Taller, P. Tsuji, C. White and J. White, ALE3D: an arbitrary Lagrangian-Eulerian multi-physics code, Technical Report LLNL-TR732040, 2017.

[30] JR. J. Pilliod AND E. PUCKETT, Second-order accurate volume-of-fluid algorithms for tracking material interfaces, J. Comput. Phys., 199 (2004), 465-502.

[31] J. J. QUIRK AND S. KARNI, On the dynamics of a shock-bubble interaction. J. Fluid Mech., 318 (1996), 129-163. 
[32] W. J. Rider AND D. B. Kothe, Reconstructing volume tracking, J. Comput. Phys., 141(2) (1998), 112-152.

[33] A. C. Robinson, T. A. Brunner, S. Carroll, R. Drake, C. J. Garasi, T. Gardiner, T. Haill, H. Hanshaw, D. Hensinger, D. Labreche, R. Lemke, E. Love, C. LUChini, S. Mosso, J. Niederhaus, C. C. Ober, S. Petney, W. J. Rider, G. Scovazzi, O. E. Strack, R. Summers, T. Trucano, V. G. Weirs, M. Wong and T. Voth, ALEGRA: an arbitrary Lagrangian-Eulerian multimaterial, multiphysics code, Technical Report AIAA 2008-1235, 2008.

[34] E. Vitali, I. N. Lomov, T. H. Antoun and D. H. Fujino, An extended Eulerian method for contacts in Godunov formulations, Internat. J. Numer. Methods Engrg., 92 (2012), 1139-1156.

[35] H. Yong, C. L. Zhai, S. JiAnG, P. SonG, Z. S. DAI AND J. F. GU, Numerical simulations of instabilities in the implosion process of Inertial Confined Fusion in $2 D$ cylindrical coordinates, Science China: Physics, Mechanics and Astronomy, 59(1) (2016), 614704-01-11. 